\title{
ON THE WEIGHTED INTEGRABILITY OF MAXIMAL ERGODIC RATIOS
}

\author{
KENNETH F. ANDERSEN
}

(Communicated by J. Marshall Ash)

\begin{abstract}
Necessary and sufficient conditions on pairs of nonnegative weight functions are given which ensure that the maximal ergodic ratios defined by means of a measure preserving conservative ergodic transformation satisfy a reverse weak type inequality. From this, weighted integrability results may be deduced.
\end{abstract}

Let $(\Omega, \Sigma, \mu)$ be a $\sigma$-finite measure space and let $T: \Omega \rightarrow \Omega$ be an ergodic, conservative measure preserving transformation. For fixed measurable $g>0$ a.e., define the maximal ergodic ratio for nonnegative $f \in L^{1}(d \mu)$ and $x \in \Omega$ by

$$
S(f, g)=\sup _{n \geq 1}\left(\frac{\sum_{j=0}^{n-1} f\left(T^{j} x\right)}{\sum_{j=0}^{n-1} g\left(T^{j} x\right)}\right)
$$

and if $\lambda>0$, set $A=\{x \in \Omega: S(f, g)>\lambda\}$. Derriennic [2] proved the reverse weak type inequality

$$
\int_{A} f v d \mu \leq \lambda \int_{A \cup T^{-1} A} \omega d \mu, \quad \mu\left(A^{c}\right)>0
$$

for the (unweighted) case $v=1, \omega=g$ and used this to deduce integrability properties of $f$ from those of $S(f, g)$. The purpose of this note is to give conditions on the nonnegative weight pairs $v, \omega$ which ensure that (1) holds. Since (1) holds for all $v, \omega$ if $\Omega$ consists of a single atom, we assume that $\Omega$ contains at least two disjoint sets of positive measure. Then our conditions characterize the pairs $v, \omega$ satisfying (1) provided $T$ satisfies the additional hypothesis $T^{-1} \Sigma=\Sigma$ (up to sets of measure zero). In particular this is the case if $T$ is invertible. Sato [5] obtained a similar characterization of a closely related inequality for invertible $T$ in the special case $g(x)=1$.

Received by the editors November 10, 1988 and, in revised form, August 15, 1989.

1980 Mathematics Subject Classification (1985 Revision). Primary 28D05.

Key words and phrases. Ergodic ratios, weighted inequalities, reverse weak type inequality, weighted integrability.

This research was supported in part by NSERC grant \#A-8185. 
Theorem. Suppose $\omega$ and $v$ are nonnegative measurable weight functions on $\Omega$. If

$$
v\left(T^{n} x\right) \leq \frac{1}{2}\left(\frac{\sum_{j=0}^{n} \omega\left(T^{j} x\right)}{\sum_{j=0}^{n} g\left(T^{j} x\right)}\right)
$$

for all $n \geq 1$ and almost all $x \in \Omega$, then (1) holds. Conversely, if $T^{-1} \Sigma=\Sigma$ and (1) holds, then

$$
v\left(T^{n} x\right) \leq 2\left(\frac{\sum_{j=0}^{n} \omega\left(T^{j} x\right)}{\sum_{j=0}^{n} g\left(T^{j} x\right)}\right)
$$

for all $n \geq 1$ and almost all $x \in \Omega$.

Corollary. If (2) holds, then

$$
\int_{\Omega}[S(f, g)+S(f, g) \circ T] \omega d \mu<\infty \text { implies } \int_{\Omega}\left[f \log ^{+}(f / g)\right] v d \mu<\infty .
$$

Proof. To prove that (2) implies (1), we first show that for each $k \geq 1$ and almost all $x \notin A$ we have

$$
\sum_{i=1}^{k} f\left(T^{i} x\right) v\left(T^{i} x\right) \leq \lambda \sum_{i=0}^{k} \omega\left(T^{i} x\right) .
$$

To see this, fix $k \geq 1$ and for $x \in \Omega$ let $\left\{\tilde{v}_{i}\right\}_{i=1}^{k}$ be the least nonincreasing majorant of $\left\{v\left(T^{i} x\right)\right\}_{i=1}^{k}$, that is,

$$
\tilde{v}_{i}(x)=\max \left\{v\left(T^{i} x\right), v\left(T^{i+1} x\right), \ldots, v\left(T^{k} x\right)\right\},
$$

and set $\tilde{v}_{0}=\tilde{v}_{1}$. Then

$$
\sum_{i=1}^{k} f\left(T^{i} x\right) v\left(T^{i} x\right) \leq \sum_{i=1}^{k} f\left(T^{i} x\right) \tilde{v}_{i}(x) \leq \sum_{i=0}^{k} f\left(T^{i} x\right) \tilde{v}_{i}(x)
$$

and summation by parts shows that the term on the right equals

$$
\left(\sum_{i=0}^{k} f\left(T^{i} x\right)\right) \tilde{v}_{k}(x)+\sum_{i=1}^{k}\left(\sum_{j=0}^{i-1} f\left(T^{j} x\right)\right)\left(\tilde{v}_{i-1}(x)-\tilde{v}_{i}(x)\right) .
$$

Now if $x \notin A, \sum_{j=0}^{i-1} f\left(T^{j} x\right) \leq \lambda \sum_{j=0}^{i-1} g\left(T^{j} x\right)$ for all $i \geq 1$, and (5) does not exceed

$\lambda\left(\sum_{i=0}^{k} g\left(T^{i} x\right)\right) \tilde{v}_{k}(x)+\sum_{i=1}^{k}\left(\lambda \sum_{j=0}^{i-1} g\left(T^{j} x\right)\right)\left(\tilde{v}_{i-1}(x)-\tilde{v}_{i}(x)\right)=\lambda \sum_{i=0}^{k} g\left(T^{i} x\right) \tilde{v}_{i}(x)$ 
again by partial summation. Now if $\left\{i_{j}\right\}_{j=1}^{m}$ denotes the points at which $\tilde{v}$ decreases, $i_{0}=-1, i_{m+1}=k$, then $\tilde{v}_{i}(x)=v\left(T^{i_{j+1}} x\right)$ for $i_{j}+1 \leq i \leq i_{j+1}$ so that

$$
\begin{aligned}
\sum_{i=0}^{k} g\left(T^{i} x\right) \tilde{v}_{i}(x) & =\sum_{j=0}^{m} \sum_{i=i_{j}+1}^{i_{j+1}} g\left(T^{i} x\right) \tilde{v}_{i}(x) \\
& =v\left(T^{i_{1}} x\right) \sum_{i=0}^{i_{1}} g\left(T^{i} x\right)+\sum_{j=1}^{m} v\left(T^{i_{j+1}} x\right) \sum_{i=i_{j}+1}^{i_{j+1}} g\left(T^{i} x\right) \\
& \leq v\left(T^{i_{1}} x\right) \sum_{i=0}^{i_{1}} g\left(T^{i} x\right)+\sum_{j=1}^{m} v\left(T^{i_{j+1}} x\right) \sum_{i=i_{j}}^{i_{j+1}} g\left(T^{i} x\right)
\end{aligned}
$$

and then since (2) shows

$$
v\left(T^{i_{1}} x\right) \leq \frac{1}{2}\left(\frac{\sum_{i=0}^{i_{1}} \omega\left(T^{i} x\right)}{\sum_{i=0}^{i_{1}} g\left(T^{i} x\right)}\right) \quad \text { and } \quad v\left(T^{i_{j+1}} x\right) \leq \frac{1}{2}\left(\frac{\sum_{i=i_{j}}^{i_{j+1}} \omega\left(T^{i} x\right)}{\sum_{i=i_{j}}^{i_{j+1}} g\left(T^{i} x\right)}\right)
$$

for $j \geq 1$, we have

$$
\sum_{i=0}^{k} g\left(T^{i} x\right) \tilde{v}_{i}(x) \leq \frac{1}{2}\left(\sum_{i=0}^{i_{1}} \omega\left(T^{i} x\right)+\sum_{j=1}^{m} \sum_{i=i_{j}}^{i_{j+1}} \omega\left(T^{i} x\right)\right) \leq \sum_{j=0}^{k} \omega\left(T^{j} x\right) .
$$

This proves (4).

Now, following Derriennic [2], since $T$ is conservative and ergodic,

$$
\begin{aligned}
\int_{A} f v d \mu & =\sum_{n=1}^{\infty} \int_{A^{c} \cap\left[\bigcap_{i=1}^{n} T^{-i} A\right]} f\left(T^{n} x\right) v\left(T^{n} x\right) d \mu \\
& =\sum_{n=1}^{\infty} \sum_{k=n}^{\infty} \int_{A^{c} \cap\left[\bigcap_{i=1}^{k} T^{-i} A\right] \cap T^{-(k+1) A^{c}}} f\left(T^{n} x\right) v\left(T^{n} x\right) d \mu \\
& =\sum_{k=1}^{\infty} \int_{A^{c} \cap\left[\bigcap_{i=1}^{k} T^{-i} A\right] \cap T^{-(k+1) A^{c}}} \sum_{n=1}^{k} f\left(T^{n} x\right) v\left(T^{n} x\right) d \mu
\end{aligned}
$$

and by (4) this does not exceed

$$
\begin{aligned}
\lambda \sum_{k=1}^{\infty} \int_{A^{c} \cap\left[\bigcap_{i=1}^{k} T^{-i} A\right] \cap T^{-(k+1)} A^{c}} \sum_{n=0}^{k} \omega\left(T^{n} x\right) d \mu \\
\quad=\lambda \sum_{k=1}^{\infty} \int_{A^{c} \cap\left[\bigcap_{i=1}^{k} T^{-i} A\right] \cap T^{-(k+1) A^{c}}}\left(\sum_{n=1}^{k} \omega\left(T^{n} x\right)+\omega(x)\right) d \mu \\
\quad=\lambda\left(\int_{A} \omega d \mu+\int_{A^{c} \cap T^{-1} A} \omega d \mu\right) \\
=\lambda \int_{A \cup T^{-1} A} \omega d \mu .
\end{aligned}
$$


This completes the proof that (2) implies (1).

To prove that (1) implies (3), fix $n \geq 1$ and a constant $c>1$. For each integer $k$ and each rational $r \in\left(c^{k}, c^{k+1}\right.$ ] let

$$
\Omega_{k, r}=\left\{x:\left(g\left(T^{n} x\right)\right)^{-1}>r \geq\left(\sum_{j=0}^{n} g\left(T^{j} x\right)\right)^{-1}>c^{k}\right\} .
$$

Since $T^{-1} \Sigma=\Sigma$, for any $F \subset \Omega_{k, r}$ with $\mu(F)>0$ there is $E_{1} \in \Sigma$ with $F=T^{-n} E_{1}$ and since $T$ is measure preserving, $\mu\left(E_{1}\right)=\mu(F)$. Then, since $T$ is ergodic, there is $E \subset E_{1}$ with $\mu(E)>0$ and $T^{-j} E, j=0, \ldots, n$ pairwise disjoint unless $\Omega=\bigcup_{j=0}^{N} T^{-j} I$ for an atom $I$ with $T^{-j} I, j=0, \ldots, N$ pairwise disjoint for some $N<n$. (see [4, Lemma 2.2; 3, Corollaries 2.12 and 2.13]).

Leaving aside the atomic case for the moment, set $f=\chi_{E}$ and $\lambda=r$. Since $T^{-n} E \subset \Omega_{k, r}$ we have

$$
S\left(\chi_{E}, g\right)\left(T^{n} x\right) \geq 1 / g\left(T^{n} x\right)>r, \quad x \in T^{-n} E
$$

so $T^{-n} E \subset T^{-n} A$, and since $T$ is measure preserving,

$$
\int_{T^{-n} E} v\left(T^{n} x\right) d \mu=\int_{T^{-n} A} \chi_{E}\left(T^{n} x\right) v\left(T^{n} x\right) d \mu=\int_{A} \chi_{E} v d \mu .
$$

On the otherhand, if $x \notin \bigcup_{j=0}^{n} T^{-j} E$ and $j_{1}<j_{2}<\cdots$ denotes those $j \geq 0$ for which $\chi_{E}\left(T^{j} x\right)=1$, then

$$
n+1 \leq j_{1}, j_{i}+n+1 \leq j_{i+1} \quad \text { and } \quad T^{j_{i}} x \in E, \quad i=1,2, \ldots
$$

so

$$
\sum_{j=1}^{j_{m}} g\left(T^{j} x\right) \geq \sum_{i=1}^{m} \sum_{j=j_{i}-n}^{j_{i}} g\left(T^{j} x\right) \geq \sum_{i=1}^{m} \frac{1}{r}=\frac{m}{r}
$$

since $T^{j_{i}-n} x \in \Omega_{k, r}$. Thus we have

$$
\frac{\sum_{j=0}^{j_{m}} \chi_{E}\left(T^{j} x\right)}{\sum_{j=0}^{j_{m}} g\left(T^{j} x\right)} \leq \frac{\sum_{j=1}^{j_{m}} \chi_{E}\left(T^{j} x\right)}{\sum_{j=1}^{j_{m}} g\left(T^{j} x\right)} \leq r
$$

which shows $S\left(\chi_{E}, g\right)\left(T^{i} x\right) \leq r$ for $i=0,1$ and $x \notin \bigcup_{j=0}^{n} T^{-j} E$. Hence $A \cup T^{-1} A \subset \bigcup_{j=0}^{n} T^{-j} E$ so

$$
\int_{A \cup T^{-1} A} \omega d \mu \leq \int_{\bigcup_{j=0}^{n} T^{-j} E} \omega d \mu=\int_{T^{-n} E}\left(\sum_{j=0}^{n} \omega\left(T^{j} x\right)\right) d \mu .
$$

Thus (1) together with (6) and (7) yield

$$
\int_{T^{-n} E} v\left(T^{n} x\right) d \mu \leq r \int_{T^{-n} E}\left(\sum_{j=0}^{n} \omega\left(T^{j} x\right)\right) d \mu .
$$


Since $F$ was arbitrary, this shows

$$
v\left(T^{n} x\right) \leq r \sum_{j=0}^{n} \omega\left(T^{j} x\right)
$$

for almost all $x \in \Omega_{k, r}$ and since $r \leq c^{k+1} \leq c\left(\sum_{j=0}^{n} g\left(T^{j} x\right)\right)^{-1}$ for such $x$, we have

$$
v\left(T^{n} x\right) \leq c\left(\frac{\sum_{j=0}^{n} \omega\left(T^{j} x\right)}{\sum_{j=0}^{n} g\left(T^{j} x\right)}\right)
$$

for almost all $x \in \Omega_{k, r}$.

Suppose now that $\Omega=\bigcup_{j=0}^{N} T^{-j} I$ for an atom $I$ with $T^{-j} I, j=0, \ldots, N$ pairwise disjoint. The argument which lead to (8) applies for $1 \leq n \leq N$. Since $T^{N+1} x=x$ for all $x \in \Omega$ in this case, if $n=m(N+1)+i, m \geq 1,0 \leq i \leq N$,

$$
\sum_{j=0}^{n} \omega\left(T^{j} x\right) \geq m \sum_{j=i+1}^{N+1+i} \omega\left(T^{j} x\right)=m \sum_{j=0}^{N} \omega\left(T^{j+1+i} x\right)
$$

and (8) for $n=N$ shows that this is greater than or equal to

$$
\begin{aligned}
c^{-1} v\left(T^{N+1+i} x\right) m \sum_{j=0}^{N} g\left(T^{j+1+i} x\right) & \geq(2 c)^{-1} v\left(T^{N+1+i} x\right) \sum_{j=0}^{n} g\left(T^{j} x\right) \\
& =(2 c)^{-1} v\left(T^{n} x\right) \sum_{j=0}^{n} g\left(T^{j} x\right) .
\end{aligned}
$$

This shows that (8) holds for all $n \geq 1$ with $c$ replaced by $2 c$.

Since $c>1$ was arbitrary and $\Omega=\bigcup_{k, r} \Omega_{k, r}$, this yields (3) and completes the proof of the Theorem.

The proof of the corollary follows exactly as in the unweighted case and, therefore, is omitted.

\section{REFERENCES}

1. K. F. Andersen and W.-S. Young, Weighted reverse weak type inequalities for the ergodic maximal function and the classes $L \log ^{+} L$, Proc. Amer. Math. Soc. 95 (1985), 275-279.

2. Y. Derriennic, On the integrability of the supremum of ergodic ratios, Ann. Probab. 1 (1973), 338-340.

3. F. J. Martin-Reyes, Inequalities for the ergodic maximal function and convergence of the averages in weighted $L^{p}$-spaces, Trans. Amer. Math. Soc. 296 (1986), 61-82.

4. F. J. Martin-Reyes and A. de la Torre, Weighted weak type inequalities for the ergodic maximal function and the pointwise ergodic theorem, Studia Math. 87 (1987), 33-56.

5. R. Sato, On a reverse maximal ergodic theorem with weights, preprint.

Department of Mathematics, University of Alberta, Edmonton, Alberta T6G 2G1, CANADA 